\title{
Clinical and Neuropsychological Factors Associated with Treatment Response and Adverse Events of Atomoxetine in Children with Attention-Deficit/ Hyperactivity Disorder
}

\author{
Kee Jeong Park and Hyo-Won Kim \\ Department of Psychiatry, Asan Medical Center, University of Ulsan College of Medicine, Seoul, Korea
}

\begin{abstract}
Objectives: The objective of this study was to investigate clinical and neuropsychological factors associated with treatment response and adverse events of atomoxetine in children with attention-deficit/hyperactivity disorder (ADHD) in Korea.

Methods: Children with ADHD were recruited at the Department of Psychiatry of Asan Medical Center from April 2015 to April 2018. Diagnoses of ADHD and comorbid psychiatric disorders were confirmed with the Kiddie-Schedule for Affective Disorders and Schizophrenia-Present and Lifetime Version. The subjects were subsequently treated with atomoxetine for 12 weeks and illness severity was scored using the ADHD Rating Scale, Clinical Global Impression-Severity scale (CGI-S) and/or Improvement scale (CGI-I), at pre- and post-treatment. They also completed the Advanced Test of Attention (ATA), while their caregivers completed the Korean Personality Rating Scale for Children (KPRC) at pre- and post-treatment. Independent t-test, Fisher's exact test, $\chi^{2}$ test, mixed betweenwithin analysis of variance and correlation analysis were used for statistical analysis.

Results: Sixty-five children with ADHD (mean age: $7.9 \pm 1.4$ years, 57 boys) were enrolled, of which, 33 (50.8\%) were treatment responders. Scores on the social dysfunction subscale of the KPRC ( $\mathrm{p}=0.021)$ and commission errors on the visual ATA ( $\mathrm{p}=0.036)$ at baseline were higher in treatment non-responders than in responders; however, the statistical significances disappeared after adjusting for multiple comparisons. Mood changes were also observed in 13 subjects (20.0\%), and three of them discontinued atomoxetine due to this. Additionally, atomoxetine-emergent mood change was observed more frequently in girls $(\mathrm{p}=0.006)$, while the intelligence quotient $(\mathrm{p}=0.040)$ was higher in those subjects with mood changes than in those without.

Conclusion: The results of our study suggest that clinical and neuropsychological factors could be associated with treatment response or adverse events of atomoxetine in children with ADHD. Further long-term studies with larger samples are needed.
\end{abstract}

Key Words: Attention-deficit/hyperactivity disorder; Atomoxetine; Treatment response; Adverse events.

Received: September 28, 2018 / Revision: January 4, 2019 / Accepted: January 9, 2019

Address for correspondence: Hyo-Won Kim, Department of Psychiatry, Asan Medical Center, University of Ulsan College of Medicine, 88 Olympic-ro 43gil, Songpa-gu, Seoul 05505, Korea

Tel: +82-2-3010-3414, Fax: +82-2-485-8381, E-mail: shingubi@amc.seoul.kr

\section{INTRODUCTION}

Attention-deficit/hyperactivity disorder (ADHD) is one of the most common neurodevelopmental disorders affecting children, with a reported worldwide prevalence in children and adolescents of 3.4\% [1]. Inattention, hyperactivity, and impulsivity constitute its core symptoms [2,3]. and children and adolescents with ADHD commonly experience behavioral problems and impaired academic and occupational achievements [3].

Both pharmacological and psychosocial treatments such

This is an Open Access article distributed under the terms of the Creative Commons Attribution Non-Commercial License (https://creativecommons.org/licenses/by-nc/4.0) which permits unrestricted non-commercial use, distribution, and reproduction in any medium, provided the original work is properly cited. as parent training and social skills training are used to reduce ADHD symptoms, whereby medication is considered to be the first line of treatment $[3,4]$. It has been reported that $75-80 \%$ of children with ADHD under medication show an improvement in key symptoms of inattention, hyperactivity, and impulsivity [5]. Medications approved for the treatment of ADHD in Korea include methylphenidate, atomoxetine, and clonidine, of which, atomoxetine is the most widely used non-stimulant medication.

The reported treatment response rate of atomoxetine in ADHD ranges between 55\% and 65\% [6,7], and its positive effects are manifested by reduction of core ADHD symptoms and an improvement of functional impairment in home and school environments [8]. However, it has been reported that 
8.4-26.0\% of children with ADHD being treated with atomoxetine discontinue medication due to non-response $[9,10]$. Therefore, identifying the predictors of treatment response to atomoxetine is necessary in order to enhance medication adherence and improve the treatment outcome. Block et al. [6] reported that the score reduction in the items "fails to give close attention or makes careless mistakes" and the "easily distracted" on ADHD Rating Scale (ARS) at the first week atomoxetine trial is a positive predictor of its treatment response. Additionally, Newcorn et al. [7] identified a certain level of improvement by the fourth week of atomoxetine treatment as a predictor of the treatment response, from six to nine weeks of atomoxetine trials. Furthermore, Treuer et al. [11] reported older age and the female sex as positive predictors of a greater remission rate with patients from nonWestern countries including Asia (China and Taiwan). However, factors associated with treatment response to atomoxetine have been studied less intensely in comparison with methylphenidate, especially with Asian population.

Adverse events of atomoxetine may include increased irritability, nausea, decreased appetite, and somnolence [12], that often results in in discontinuation of treatment $[10,13]$. However, there has been little research on the predictive factors of adverse events of atomoxetine. Identification of these predictors of adverse events can contribute to enhancing the medication adherence of atomoxetine and decreasing the time required to achieve the desired therapeutic goals, thus minimizing the loss incurred to individual patients and society caused by non-response and treatment delay.

This study aims to investigate the clinical and neuropsychological factors associated with treatment response and adverse events of atomoxetine in Korean children with ADHD.

\section{METHODS}

\section{Subjects and study design}

Subjects were recruited at the Department of Psychiatry of Asan Medical Center from April 2015 to April 2018. The inclusion criteria for this study included the following: 1) Children aged 5-12 years; 2) Diagnosis of ADHD under the Diagnostic and Statistical Manual of Mental DisordersFourth Edition (DSM-IV) [14] and Kiddie-Schedule for Affective Disorders and Schizophrenia-Present and Lifetime Version (K-SADS-PL) [15]; and 3) Started on atomoxetine. The exclusion criteria included the following: 1) Presence of learning disorders, mental retardation, bipolar disorder, psychotic disorders, developmental disorders, organic brain disease, epilepsy, or neurological disorders; 2) Presence of tic disorders, obsessive-compulsive disorder, major depressive disorder, or anxiety disorders that required pharmaco- therapy; 3) Presence of severe suicidal ideation; 4) History of methylphenidate or atomoxetine treatment within the past six months; 5) Current serious medical conditions (such as cardiovascular, hepatic, renal, and respiratory disorders, and glaucoma); and 6) Current medication of alpha-2 adrenergic receptor agonists, antidepressants, antipsychotics, benzodiazepines, and anticonvulsants or dietary supplements with significant effects on the central nervous system.

Prior to initiating the atomoxetine medication, the subjects underwent the following baseline pre-treatment tests: The ARS [16], Advanced Test of Attention (ATA) [17], and Clinical Global Impression-Severity scale (CGI-S) [18], while their caregivers underwent the Korean Personality Rating Scale for Children (KPRC) [19]. After completion of the 12 weeks of atomoxetine treatment, the subjects underwent the following post-treatment tests: all the aforementioned baseline tests, as well as the Clinical Global Impression-Improvement scale (CGI-I) [18] for the assessment of treatment response. Among the subjects who withdrew from the study without completing the 12 weeks atomoxetine treatment, those who took the medication at least once were included in the analysis.

Standard-dose atomoxetine treatment was conducted in compliance with the following guidelines, whereby the optimal dose was determined based on its clinical efficacy as judged by the clinician. The initial dose of atomoxetine (Strattera $^{\circledR}$, Eli Lilly and Company, Indianapolis, IN, USA) was set at $0.5 \mathrm{mg} / \mathrm{kg} / \mathrm{day}$, with the maximum dose at $1.4 \mathrm{mg} / \mathrm{kg} /$ day. Dose adjustment was performed at intervals not shorter than a week. Subjects with a CGI-I score of 2 or less, or a decrease in the ARS total score of $\geq 50 \%$ from the baseline after 12 weeks atomoxetine treatment were classified as treatment responders. For those subjects who had withdrawn from the study prior to completing the 12 weeks atomoxetine treatment, treatment response was assessed based on the CGI-I and ARS scores at the time of withdrawal.

Adverse events were also evaluated, using a checklist that was partially modified from the 61-item checklist presented in a study with methylphenidate [20], this checklist was administered by a clinician as follows: each of the 44 types of adverse events was evaluated based on severity (mild, moderate, or severe), causality (not related, doubtful, possible, probable, or very likely), and clinical outcomes (resolved, improved, no change, aggravated, or serious adverse events), and the onset and resolution dates were registered. Furthermore, this checklist was administered at baseline (pre-treatment) and 12th week (post-treatment), and during this period, the manifestations of six mood states (depressed mood, labile affect, irritability, anger/hostility, euphoria, and loss of interest) were assessed based on severity and causality, and 
those with any evaluation of severity (mild, moderate, or severe) and a causality of possible, probable, or very likely were defined as "mood-related adverse events" associated with the use of atomoxetine.

This study was approved by the Institutional Review Board of Asan Medical Center (IRB NO. 2014-0157), and written consent for the overall study procedure was obtained from all the participants and their caregivers.

\section{Assessment tools}

Kiddie-Schedule for Affective Disorders and SchizophreniaPresent and Lifetime Version (K-SADS-PL) [15]

The K-SADS-PL is a semi-structured interview tool designed to assess the severity of the current and lifetime morbidity of 32 DSM-IV child and adolescent psychiatric disorders. Its reliability and validity has been verified [15]. Additionally, the reliability and validity of the Korean version of K-SADSPL, translated by Kim et al. [21], has been studied with respect to the items related to ADHD, tic disorders, oppositional defiant disorder, depressive disorders, and anxiety disorders. In this study, K-SADS-PL was administered by a pediatric psychiatrist and a clinical psychologist experienced in using the tool and familiar with clinical interviews.

\section{Intelligence tests}

Korean-Wechsler Preschool and Primary Scale of Intelligence (K-WPPSI) [22]

The Korean-Wechsler Preschool and Primary Scale of Intelligence (K-WPPSI) is an individually administered intelligence test for children aged 3 years 0 months to 7 years 3 months. It is the K-WPPSI adapted for the Korean population and has been standardized by the Korean Institute of Developmental Tests. As an intelligence test developed to measure the intelligence of preschoolers and early schoolage children of ages lower than the defined age range for Wechsler Intelligence Scale for Children (WISC), it consists of two subscales: Verbal Intelligence Quotient (VIQ) and Performance Intelligence Quotient (PIQ), with each subscale comprising of six subtests. The overall test result is presented as the Full Scale Intelligence Quotient (FSIQ) [22].

Korean-Wechsler Intelligence Scale for Children-Third Edition (K-WISC-III) [22]

The Korean-Wechsler Intelligence Scale for ChildrenThird Edition (K-WISC-III) is an individually administered intelligence test for the clinical assessment of cognitive abilities for children aged 6 years 0 months to 16 years 11 months. It is the KWISC-III adapted for the Korean population and has been standardized by the Korean Institute for Developmental Tests. The K-WISC-III assesses cognitive abilities with a variety of subtests designed to measure specific abilities. In addition to the VIQ, PIQ, and FSIQ, it provides fourfactor index scores based on factor analysis. Unlike its previous versions, this version consists of 13 subtests, with the subtest "symbol search" added to the standard 12 subtests for testing children's cognitive abilities [23].

Korean-Wechsler Intelligence Scale for Children-Fourth Edition (K-WISC-IV) [24]

The K-WISC Fourth Edition (K-WISC-IV), a revised version of K-WISC-III (2001), is an intelligence test for children and adolescents aged 6 years 0 months to 16 years 11 months. K-WISC-IV provides not only the FSIQ, which indicates the overall cognitive abilities, but also subtest and index scores that indicate specific cognitive profiles. This version consists of five supplementary subtests (picture completion, letternumber sequencing, matrix reasoning, word reasoning, and cancellation), comprising a total of 15 subtests, of which the results are presented as the FSIQ and four index scores. The terms, VIQ and PIQ are replaced with verbal comprehension index and perceptual reasoning index, respectively [24].

\section{ADHD Rating Scale (ARS)}

The ARS is an 18-item scale developed by DuPaul [16] that is used to rate ADHD symptoms in school-age children. It is designed to be evaluated by researchers, parents, and teachers and presented as a parent-report or teacher-report inventory. The validity and reliability of this scale has been verified in many studies; So et al. [25] have verified the validity and reliability of the Korean version of the parent-report and teacher-report ARS with 1044 children.

\section{Advanced Test of Attention (ATA)}

The ATA is a computerized continuous performance test for the quantitative assessment of children's attention and impulse control abilities. It has been developed and standardized for Korean children by Shin et al. [17], and consists of a visual test and a auditory test, each yielding four indices: omission errors, commission errors, mean response time, and response time variability (standard deviation of reaction time). In this study, the z-score was used, whereby a score of 1.5 or more was determined as deviating from the normal range and the $1.0-1.5$ as the boundary conditions. The internal consistency coefficient of the ATA is 0.87 .

\section{Korean Personality Rating Scale for Children (KPRC)}

The KPRC was developed by modifying and complementing the Korean Personality Inventory for Children (KPI-C) 
[26] in order to address the problems of the latter, and has been standardized for children aged 3 to 17 years [19]. It consists of 177 items rated on a 4-point Likert scale that are categorized into three validity scales (T-R scale, L scale, and F scale), one ego-resilience scale, and 10 clinical scales (verbal development, physical development, anxiety, depression, somatic concern, delinquency, hyperactivity, family dysfunction, social dysfunction, and psychoticism).

\section{Clinical Global Impression-Improvement and Severity scales (CGI-I/S)}

The CGI-I/S is an observer-rated scale developed by Guy [18]. This scale used to describe the illness severity, response to treatment, and course of treatment, and is widely used in clinical studies as it can be administered quickly and easily used for evaluating psychiatric disorders. Many studies have demonstrated that it has a sufficiently high validity despite being used by raters without a sufficient level of knowledge of the clinical manifestations of these disorders. The CGI-I is designed to rate improvement on a 7-point Likert scale (1= very much improved; 7=very much worse), while the CGI-S is designed to rate the current severity on a 7-point Likert scale (1=normal; $7=$ extremely ill).

\section{Data analysis}

Five of the recruited subjects withdrew from the study prior to 12 th week, and their data were analyzed using the Last Observation Carried Forward method. The collected data were analyzed as follows: $\chi^{2}$ test or Fisher's exact test to compare the categorical variables, and independent sample t-test to compare the continuous variables; Bonferroni correction to adjust for multiple comparisons; Paired t-test to compare pre- and post-treatment clinical variables, and mixed between-within analysis of variance to compare pre- and posttreatment clinical variables between the treatment responder and non-responder groups; Correlation analysis to assess the correlations between individual clinical variable pairs representing pre- and post-treatment changes. Statistical analysis was performed using IBM SPSS 22 Statistics for Windows (IBM Corp., Armonk, NY, USA). The significance level was set at $\mathrm{p}<0.05$.

\section{RESULTS}

Sixty-seven children with ADHD (mean age: $7.9 \pm 1.4$ years; 58 boys) were recruited from April 2015 to April 2018, of which 65 children (mean age: $7.9 \pm 1.4$ years; 57 boys) who were treated with atomoxetine at least once were enrolled in the study. Of these subjects, five discontinued the atomoxetine trial prior to 12 th week for reasons of refusal $(n=1)$, adverse events $(n=3)$, and a concurrent medication of antipsychotics $(n=1)$.

\section{Demographic characteristics}

In this study, boys were the majority compared to girls [57 $(87.7 \%)$ vs. 8 (12.3\%)]. The overall mean FSIQ score was $94.1 \pm 11.5$, and the subjects were grouped as follows: combined type $(\mathrm{n}=34,52.3 \%)$, predominantly inattentive type ( $n=27,41.5 \%)$, predominantly hyperactive-impulsive type $(n=3,4.6 \%)$, and not otherwise specified $(n=1,1.5 \%)$. The most prevalent comorbid disorder observed was oppositional defiant disorder and conduct disorder ( $n=9,13.8 \%)$, followed by tic disorders $(n=9,13.8 \%)$, anxiety disorders $(n=2$, $3.1 \%)$, and enuresis $(n=1,1.5 \%)$.

The overall mean atomoxetine dose was $27.2 \pm 9.7 \mathrm{mg} /$ day and $0.89 \pm 0.23 \mathrm{mg} / \mathrm{kg} /$ day on average. The mean post-treatment CGI-S score decreased by $1.2 \pm 0.9$ on average compared to the pre-treatment (baseline) score.

No significant differences were observed in age, sex, FSIQ, age of onset, and ADHD subtype between the treatment dropout group ( $n=5$, treatment $<12$ weeks) and the treatment retention group ( $n=60$, treatment $=12$ weeks). With the exception of anxiety disorder, which affected a significantly higher proportion in the treatment dropout group compared to the treatment retention group $\left(1 / 5\right.$ vs. $\left.1 / 60 ; \chi^{2}=5.202, p=0.023\right)$, no significant differences were observed in comorbid disorders. Additionally, the treatment dropout group was administered a significantly lower mean atomoxetine dose compared with the treatment retention group $(0.22 \pm 0.24 \mathrm{mg} /$ $\mathrm{kg} /$ day vs. $0.50 \pm 1.39 \mathrm{mg} / \mathrm{kg} /$ day; $\mathrm{U}=54.000, \mathrm{p}=0.018$ ).

Subjects with a CGI-I score of 2 or less, or a decrease in the ARS total score of $\geq 50 \%$ from baseline to 12 weeks atomoxetine treatment were classified as treatment responders; hence, 33 out of the 65 subjects (50.8\%) were treatment responders. No significant differences were observed in age, sex, FSIQ, ADHD age of onset, atomoxetine dose, ADHD subtype, and comorbid disorders between the treatment responder and non-responder groups (Table 1).

\section{Comparison between the atomoxetine treatment responder and non-responder groups}

The pre- and post-treatment comparisons of the ARS scores between the treatment responder and non-responder groups revealed significant differences in the "inattentive" subscale with respect to the main effect for time $[\mathrm{F}(1,62)=$ 105.263, $\mathrm{p}<0.001]$, main effect for group $[\mathrm{F}(1,62)=4.824, \mathrm{p}=$ $0.032]$, and time-by-group interaction effect $[\mathrm{F}(1,62)=17.588$, $\mathrm{p}<0.001]$. In the "hyperactive-impulsive" subscale, significant differences were observed in the main effect for time $[\mathrm{F}(1$, $62)=55.403, p<0.001]$ and the time-by-group interaction effect $[F(1,62)=14.247, \mathrm{p}<0.001]$, however, no significant differ- 
Table 1. Comparison of demographic characteristics between responders and non-responders to atomoxetine

\begin{tabular}{|c|c|c|c|c|}
\hline & Responders $(\mathrm{n}=33)$ & Non-responders $(\mathrm{n}=32)$ & t or $\chi^{2}$ & $\mathrm{p}$ \\
\hline Age, mean (SD) & $7.9(1.6)$ & $7.8(1.3)$ & 0.524 & 0.602 \\
\hline Sex, boys, n (\%) & $28(84.8)$ & $29(90.6)$ & & $0.708^{*}$ \\
\hline $\mathrm{FSIQ}$ & $94.5(10.5)$ & $93.8(12.6)$ & 0.245 & 0.807 \\
\hline Age of onset, mean (SD) & $5.1(1.5)$ & $5.7(1.7)$ & -1.359 & 0.179 \\
\hline Medication dose, mg/kg/day, mean (SD) & $0.90(0.24)$ & $0.87(0.21)$ & 0.580 & 0.564 \\
\hline ADHD subtype, n (\%) & & & 2.362 & 0.501 \\
\hline Inattentive & $16(48.5)$ & $11(34.4)$ & & \\
\hline Hyperactive-impulsive & $1(3.0)$ & $2(6.3)$ & & \\
\hline Combined & $16(48.5)$ & $18(56.3)$ & & \\
\hline NOS & $0(0.0)$ & $1(3.1)$ & & \\
\hline \multicolumn{5}{|l|}{ Comorbid disorder, n (\%) } \\
\hline ODD/CD & $5(15.2)$ & $4(12.5)$ & & $1.000 *$ \\
\hline Anxiety disorder & $0(0.0)$ & $2(6.3)$ & & $0.239 *$ \\
\hline Tic disorder & $5(15.2)$ & $4(12.5)$ & & $1.000 *$ \\
\hline Enuresis & $1(3.0)$ & $0(0.0)$ & & $1.000 *$ \\
\hline
\end{tabular}

Definition of response to atomoxetine was determined as reduction in ADHD Rating Scale score of more than $50 \%$ between pretreatment and post-treatment (12th week) or less than three in Clinical Global Impression-Improvement scale score at post-treatment (12th week). *using Fisher's exact test. ADHD: attention-deficit/hyperactivity disorder, CD: conduct disorder, FSIQ: Full-Scale Intelligence Quotient, NOS: not otherwise specified, ODD: oppositional defiant disorder, SD: standard deviation

ence was observed in the main effect for group $[\mathrm{F}(1,62)=1.028$, $\mathrm{p}=0.315]$. The pre- and post-treatment comparisons of the CGI-S scores between the treatment responder and non-responder groups also revealed significant differences in the main effect for time $[F(1,62)=166.100, p<0.001]$, main effect for group $[\mathrm{F}(1,62)=8.447, \mathrm{p}=0.005]$, and time-by-group interaction effect $[\mathrm{F}(1,62)=33.243, \mathrm{p}<0.001]$.

No significant intergroup differences between the treatment responder and non-responder groups were observed in the pre-treatment scores of both subscales of the ARS (inattentive and hyperactive-impulsive) as well as the CGI-S (Table 2)

With respect to the KPRC, the mean pre-treatment score of the non-responder group was significantly higher in the "social dysfunction" subscale $(\mathrm{t}=-2.367, \mathrm{p}=0.021)$; however, the statistical significance disappeared after the post-hoc test. No significant intergroup differences were observed in the remaining 10 subscales (Table 2 ).

With respect to the ATA, the mean pre-treatment score of the non-responder group was significantly higher in "commission errors" on the visual ATA ( $\mathrm{t}=-2.140, \mathrm{p}=0.036)$; however, the statistical difference disappeared after the post-hoc test. No significant intergroup differences were observed in all the other subscales of the ATA (Table 2).

\section{Adverse events of atomoxetine}

The Treatment-Emergent Adverse Events (TEAEs) most frequently observed in this study were stomach-related TEAEs $(n=38,58.5 \%)$, followed by those related to sleep $(n=26,40 \%)$, $\operatorname{mood}(\mathrm{n}=13,20 \%)$, and cardiovascular symptoms $(\mathrm{n}=8$, $12.3 \%)$. Other TEAEs observed included headache $(n=11$, $16.9 \%)$, anxiety $(n=5,7.7 \%)$, dizziness $(n=5,7.7 \%)$, nervousness $(n=4,6.2 \%)$, and sweating $(n=1,1.5 \%)$ (Table 3$)$.

\section{Comparison of demographic characteristics between the subjects with and without Atomoxetine-Emergent Mood Change (AEMC)}

Atomoxetine-Emergent Mood Change (AEMC) includes the following states: depressed mood, labile affect, irritability, anger/hostility, euphoria, and loss of interest. It was observed in 13 subjects (20.0\%), of which six experienced two or more mood-related adverse events and three discontinued atomoxetine medication due to these events. The most frequently observed mood-related adverse event was depressed mood $(n=6,9.2 \%)$, followed by irritability $(n=5,7.7 \%)$, anger/hostility $(n=5,7.7 \%)$, labile affect $(n=5,7.7 \%)$, and loss of interest $(n=1,1.5 \%)$.

No significant intergroup differences were observed in the age, ADHD subtype, and comorbid disorders between the AEMC and non-AEMC groups. Furthermore, the AEMC group had a higher proportion of girls $(\mathrm{p}=0.006)$ and a significantly higher mean FSIQ $(\mathrm{p}=0.040)$ (Table 4). However, the statistical significance of both intergroup differences disappeared after the post-hoc test (critical p-value after Bonferroni correction, 0.00625).

No significant intergroup differences between the AEMC and non-AEMC groups were observed in the pre-treatment scores of all subscales of the ARS, KPRC, ATA, and CGI-S. 
Table 2. Comparison of clinical and neuropsychological characteristics between responders and non-responders to atomoxetine

\begin{tabular}{|c|c|c|c|c|}
\hline & Responders $(n=33)$ & Non-responders $(n=32)$ & $t$ & \\
\hline & Mean (SD) & Mean (SD) & $T$ & $P$ \\
\hline \multicolumn{5}{|l|}{ ARS (pre-treatment) } \\
\hline Inattentive & $17.5(3.4)$ & $17.5(4.4)$ & -0.046 & 0.963 \\
\hline Hyperactive-impulsive & $12.6(4.9)$ & $12.0(5.8)$ & 0.412 & 0.682 \\
\hline CGI-S (pre-treatment) & $5.2(0.6)$ & $5.1(1.0)$ & 0.284 & 0.778 \\
\hline \multicolumn{5}{|l|}{ KPRC (pre-treatment) } \\
\hline Ego-resilience & $47.4(13.5)$ & $46.0(11.8)$ & 0.453 & 0.652 \\
\hline Verbal development & $54.9(12.8)$ & $55.3(9.8)$ & -0.110 & 0.913 \\
\hline Physical development & $55.0(13.0)$ & $53.9(8.7)$ & 0.398 & 0.692 \\
\hline Anxiety & $50.4(9.3)$ & $50.4(11.5)$ & -0.005 & 0.996 \\
\hline Depression & $52.0(10.4)$ & $54.7(9.7)$ & -1.073 & 0.287 \\
\hline Somatic concern & $44.9(8.5)$ & $47.3(6.9)$ & -1.285 & 0.203 \\
\hline Delinquency & $56.9(11.8)$ & $59.8(10.8)$ & -1.058 & 0.294 \\
\hline Hyperactivity & $63.0(9.5)$ & $62.0(12.1)$ & 0.395 & 0.694 \\
\hline Family dysfunction & $52.4(13.4)$ & $54.5(14.3)$ & -0.595 & 0.554 \\
\hline Social dysfunction & $47.8(8.6)$ & $53.4(10.3)$ & -2.367 & $0.021^{*}$ \\
\hline Psychoticism & $56.7(12.2)$ & $57.3(12.4)$ & -0.221 & 0.825 \\
\hline \multicolumn{5}{|l|}{ ATA (pre-treatment) } \\
\hline Omission errors, visual & $5.1(7.1)$ & $4.8(3.9)$ & 0.201 & 0.841 \\
\hline Commission errors, visual & $3.0(3.4)$ & $5.0(3.8)$ & -2.140 & $0.036^{*}$ \\
\hline Reaction time, visual & $1.5(1.9)$ & $0.7(1.5)$ & 1.875 & 0.065 \\
\hline Reaction time variability, visual & $3.4(4.4)$ & $3.1(2.3)$ & 0.383 & 0.703 \\
\hline Omission errors, auditory & $1.9(2.7)$ & $1.4(1.9)$ & 0.805 & 0.424 \\
\hline Commission errors, auditory & $2.1(2.3)$ & $2.5(3.2)$ & -0.614 & 0.542 \\
\hline Reaction time, auditory & $-0.5(1.3)$ & $-2.2(6.5)$ & 1.447 & 0.153 \\
\hline Reaction time variability, auditory & $0.3(1.4)$ & $0.1(1.1)$ & 0.361 & 0.720 \\
\hline
\end{tabular}

Definition of response to atomoxetine was determined as reduction in ARS score of more than $50 \%$ between pre-treatment and post-treatment (12th week) or less than three in Clinical Global Impression-Improvement scale score at post-treatment (12th week). ${ }^{*} p<0.05$. ARS: attention-deficit/hyperactivity disorder Rating Scale, ATA: Advanced Test of Attention, CGI-S: Clinical Global Impression-Severity scale, KPRC: Korean Personality Rating scale for Children, SD: standard deviation

\section{Correlation coefficients between the subscales of the KPRC and other ADHD-related scales in terms of pre- and post-treatment changes}

Change was calculated by subtracting from post-treatment (12th week) measurement to pre-treatment measurement. The changes in the CGI-S score showed correlations with none of the changes in the KPRC subscale score; however, the changes in the "inattentive" subscale of ARS showed significant positive correlations with the changes in the KPRC subscales of depression $(r=0.263, p=0.039)$, delinquency $(r=$ $0.293, \mathrm{p}=0.021)$, family dysfunction $(\mathrm{r}=0.294, \mathrm{p}=0.020)$, and psychoticism ( $r=0.270, p=0.034)$. Additionally, the changes in the "hyperactive-impulsive" subscale of ARS were also positively correlated with the changes in the KPRC subscales of delinquency $(\mathrm{r}=0.332, \mathrm{p}=0.008)$, hyperactivity $(\mathrm{r}=0.358$, $\mathrm{p}=0.004)$, family dysfunction $(\mathrm{r}=0.358, \mathrm{p}=0.004)$, and $\mathrm{psy}$ choticism $(\mathrm{r}=0.295, \mathrm{p}=0.020)$ (Table 5).

\section{DISCUSSION}

In this study, atomoxetine treatment non-responders showed higher baseline test scores in the "social dysfunction" subscale of the KPRC and the "commission errors" of the visual ATA, suggesting that these two subscales could be factors associated with atomoxetine treatment response. Moreover, sex and Intelligence Quotient (IQ) were observed as factors associated with AEMC. Finally, the AEMC group had a higher proportion of girls and a higher mean FSIQ as compared to the non-AEMC group.

The treatment non-response rate of this study (49.2\%) was similar to those presented in previous studies. Newcorn et al. [7] defined non-response to treatment as less than $40 \%$ decline in ARS scores after a short-term atomoxetine treatment (6 to 9 weeks), compared to the baseline value. The non-response rate of atomoxetine treatment in their study was reported to be $40 \%$, however, in contrast to this, in long-term 
outcomes of atomoxetine treatment, treatment non-response rates of $13 \%$ and $10 \%$ were reported after 6 and 24 months of treatment, respectively. [27].

Schwartz and Correll [8] reported a bimodal pattern of treatment response to atomoxetine and suggested that there is a need to examine the factors associated with the genotype or endophenotype of treatment non-responders. In this study, the atomoxetine treatment responders showed significantly lower social dysfunction scores and commission errors scores in the KPRC and the visual ATA than did the nonresponers, respectively [28]. Mentions of a social function or

Table 3. Treatment-emergent adverse events that occurred in $\geq$ $5 \%$ of subjects

\begin{tabular}{lc}
\hline \multicolumn{1}{c}{ MedDRA preferred term } & Incidence, $\mathrm{n}(\%)$ \\
\hline Nausea/vomiting & $23(35.4)$ \\
Decreased appetite & $21(32.3)$ \\
Trouble falling asleep & $18(27.7)$ \\
Headache & $11(16.9)$ \\
Somnolence & $8(12.3)$ \\
Stomach discomfort & $7(10.8)$ \\
Sleep maintenance insomnia & $7(10.8)$ \\
Fatigue & $7(10.8)$ \\
Depressed mood & $6(9.2)$ \\
Irritability & $5(7.7)$ \\
Anger/hostility & $5(7.7)$ \\
Labile affect & $5(7.7)$ \\
Increased appetite & $5(7.7)$ \\
Anxiety & $5(7.7)$ \\
Dizziness & $5(7.7)$ \\
Abdominal pain & $4(6.2)$ \\
Nervousness & $4(6.2)$ \\
\hline MedDRA: Medical dictionary for regulatory activities
\end{tabular}

MedDRA: Medical dictionary for regulatory activities continuous performance test have rarely been made in previous studies on predictors of treatment response to atomoxetine. The aforementioned results of this study are consistent with the report of a Korean study [28] that revealed that methylphenidate treatment responders showed a significantly smaller response time variability (standard deviation of reaction time) in the baseline visual ATA. Based on this, it could be inferred that social dysfunction in the KPRC and commission errors in the visual ATA are not atomoxetinespecific predictors, but could serve as predictors of treatment outcomes for general ADHD medication exposure in Korean children.

Furthermore, significant intergroup differences in sex and FSIQ were also observed between the AEMC group $(n=13$, 20.0\%) and non-AEMC group. AEMCs were observed more frequently in girls, presumably because they show easily observable subtle mood changes while concurrently showing impulsivity and hyperactivity less frequently, and further tend to report mood-related adverse events more frequently as compared to boys, on the account of their earlier onset of puberty. Similarly, girls were found to show mood-related adverse events more frequently than boys in a study on antidepressants [29]. Whereas many studies have reported on the association between higher IQ and better treatment response $[30,31]$ and outcome $[32,33]$ in ADHD children, there are no reports on the association between AEMC and IQ [3436]. The effects of IQ on AEMC would have to be elucidated in a future study.

Little difference was observed in the atomoxetine dose administered between the AEMC and non-AEMC groups in this study. This allows for the assumption that in a clinical population that is likely to show an effective treatment out-

Table 4. Comparison of demographic characteristics between participants with and without AEMC

\begin{tabular}{|c|c|c|c|c|}
\hline & $\operatorname{AEMC~}(n=13)$ & Non-AEMC $(n=52)$ & tor $\chi^{2}$ & $p$ \\
\hline Age, mean (SD) & $8.2(1.5)$ & $7.8(1.4)$ & 1.071 & 0.288 \\
\hline Sex, boys, n (\%) & $8(61.5)$ & $49(94.2)$ & & $0.006^{* \dagger}$ \\
\hline FSIQ & $99.9(7.8)$ & $92.7(11.8)$ & 2.094 & $0.040^{* \dagger}$ \\
\hline ADHD subtype, n (\%) & & & 0.784 & 0.853 \\
\hline Inattentive & $6(46.2)$ & $21(40.4)$ & & \\
\hline Hyperactive-impulsive & $1(7.7)$ & $2(3.8)$ & & \\
\hline Combined & $6(46.2)$ & $28(53.8)$ & & \\
\hline NOS & $0(0.0)$ & $1(1.9)$ & & \\
\hline \multicolumn{5}{|l|}{ Comorbid disorder, n (\%) } \\
\hline ODD/CD & $2(15.4)$ & $7(13.5)$ & & $1.000^{*}$ \\
\hline Anxiety disorder & $0(0.0)$ & $2(3.8)$ & & $1.000^{*}$ \\
\hline Tic disorder & $0(0.0)$ & $1(1.9)$ & & $1.000^{*}$ \\
\hline Enuresis & $1(7.7)$ & $8(15.4)$ & & $0.674^{*}$ \\
\hline
\end{tabular}

*using Fisher's exact test, ${ }^{\dagger} \mathrm{p}<0.05$. ADHD: attention-deficit/hyperactivity disorder, AEMC: Atomoxetine-Emergent Mood Change, CD: conduct disorder, FSIQ: Full-Scale Intelligence Quotient, NOS: not otherwise specified, ODD: oppositional defiant disorder, SD: standard deviation 
Table 5. Correlation coefficients between KPRC changes and $A D H D$ related measures changes

\begin{tabular}{lrcc}
\hline \multirow{2}{*}{ KPRC changes } & CGI-S & \multicolumn{2}{c}{ ARS changes } \\
\cline { 3 - 4 } changes & Inattentive & $\begin{array}{c}\text { Hyperactive- } \\
\text { impulsive }\end{array}$ \\
\hline Ego resilience & -0.011 & -0.108 & -0.159 \\
Verbal development & 0.051 & 0.202 & 0.199 \\
Physical development & -0.019 & 0.173 & 0.102 \\
Anxiety & 0.161 & 0.202 & 0.197 \\
Depression & 0.020 & $0.263^{*}$ & 0.102 \\
Somatic concern & 0.112 & 0.119 & 0.173 \\
Delinquency & 0.238 & $0.293^{*}$ & $0.332^{*}$ \\
Hyperactivity & 0.204 & 0.240 & $0.322^{\dagger}$ \\
Family dysfunction & 0.117 & $0.294^{*}$ & $0.358^{\dagger}$ \\
Social dysfunction & -0.113 & 0.107 & 0.099 \\
Psychoticism & 0.183 & $0.270^{*}$ & $0.295^{*}$ \\
\hline
\end{tabular}

Change was calculated by subtracting from post-treatment (12th week) measurement to pre-treatment measurement. ${ }^{*} p<0.05$, ${ }^{\dagger} p<0.01$. ADHD: attention-deficit/hyperactivity disorder, ARS: attention-deficit/hyperactivity disorder Rating Scale, CGI-S: Clinical Global Impression-Severity scale, KPRC: Korean Personality Rating Scale for Children

come at a lower dose of atomoxetine, an unnecessarily relatively higher dose can act as a factor that boosts the occurrence of the adverse events. However, considering the reports of previous studies on the common adverse events of atomoxetine, the occurrence of these adverse events is not dosedependent [37], and is reportedly positively correlated with CYP2D6 metabolic activity [38], the individual drug metabolizing ability or drug sensitivity that may have influenced the occurrence of these adverse events in addition to atomoxetine dosing.

After the 12 weeks atomoxetine treatment, positive correlations were observed between the decrease in the score of the ARS "inattentive" subscale and the decrease in the scores of the KPRC subscales of depression, delinquency, family dysfunction, and psychoticism. Similarly, in the ARS "hyperactive-impulsive" subscale, the post-treatment decrease was positively correlated with the decreased KPRC subscales of delinquency, hyperactivity, family dysfunction, and psychoticism. The result of the KPRC subscale items being positively correlated with each of the ARS subscales is similar to an earlier research finding [39], as well as to that of a previous study [40], which reported a significant correlation between reduced ADHD symptoms and functional improvement after ADHD pharmacotherapy.

This study has several limitations. First, the sample size was small and analysis was performed based on observations made during a short period (3 months). Second, as an openlabel study performed with patients at only one university hospital, these results may not reflect the characteristics of the entire ADHD population. Third, this study did not control for non-pharmacological treatments that could potentially affect the treatment response in ADHD children. Fourth, there was a significant difference in the comorbidity of anxiety disorders between the treatment dropout group and treatment retention group, with 1 out of 5 and 1 out of 60 subjects, respectively, which could be assumed to have had little impact on the actual outcome, but should nevertheless be considered in the interpretation of the results of this study.

\section{CONCLUSION}

The results of this study suggest a potential association between the clinical and neuropsychological factors, and the treatment response or adverse events of atomoxetine in Korean ADHD children. This association would have to be verified through further long-term studies with larger sample sizes and more detailed analyses.

\section{Acknowledgments}

This study was supported by a grant from the National Research Foundation (NRF) of Korea funded by the Ministry of Science and Information \& Communication Technology (ICT) as part of the 2015 Basic Research Program (No.2015R1A1A3A04001349).

\section{Conflicts of Interest}

The authors have no potential conflicts of interest to disclose.

\section{REFERENCES}

1) Polanczyk GV, Salum GA, Sugaya LS, Caye A, Rohde LA. Annual research review: a meta-analysis of the worldwide prevalence of mental disorders in children and adolescents. J Child Psychol Psychiatry 2015;56:345-365.

2) Volk HE, Neuman RJ, Todd RD. A systematic evaluation of ADHD and comorbid psychopathology in a population-based twin sample. J Am Acad Child Adolesc Psychiatry 2005;44:768-775.

3) Barkley RA. ADHD in adults: developmental course and outcome of children with ADHD, and ADHD in clinic referred adults. In: Barkley RA, editor. Attention-deficit hyperactivity disorder: a handbook for diagnosis and treatment. 3rd ed. New York: The Guilford Press;2006. p.248-296.

4) Pliszka SR, Crismon ML, Hughes CW, Corners CK, Emslie GJ, Jensen PS, et al. The Texas children's medication algorithm project: revision of the algorithm for pharmacotherapy of attention-deficit/ hyperactivity disorder. J Am Acad Child Adolesc Psychiatry 2006; 45:642-657.

5) Briars L, Todd T. A review of pharmacological management of attention-deficit/hyperactivity disorder. J Pediatr Pharmacol Ther 2016;21:192-206.

6) Block SL, Williams D, Donnelly CL, Dunn DW, Saylor KE, Ruberg SJ. Post hoc analysis: early changes in ADHD-RS items predict longer term response to atomoxetine in pediatric patients. Clin Pediatr (Phila) 2010;49:768-776.

7) Newcorn JH, Sutton VK, Weiss MD, Sumner CR. Clinical responses to atomoxetine in attention-deficit/hyperactivity disorder: the integrated data exploratory analysis (IDEA) study. J Am Acad Child Adolesc Psychiatry 2009;48:511-518.

8) Schwartz S, Correll CU. Efficacy and safety of atomoxetine in chil- 
dren and adolescents with attention-deficit/hyperactivity disorder: results from a comprehensive meta-analysis and metaregression. J Am Acad Child Adolesc Psychiatry 2014;53:174-187.

9) Durell TM, Pumariega AJ, Rothe EM, Tamayo JM, Baron D, Williams D. Effects of open-label atomoxetine on African-American and Caucasian pediatric outpatients with attention-deficit/hyperactivity disorder. Ann Clin Psychiatry 2009;21:26-37.

10) Torres A, Whitney J, Rao S, Tilley C, Lobel R, Gonzalez-Heydrich J. Tolerability of atomoxetine for treatment of pediatric attentiondeficit/hyperactivity disorder in the context of epilepsy. Epilepsy Behav 2011;20:95-102.

11) Treuer $T$, Feng $Q$, Desaiah $D$, Altin $M, W u$, El-Shafei A, et al. Predictors of pharmacological treatment outcomes with atomoxetine or methylphenidate in patients with attention-deficit/hyperactivity disorder from China, Egypt, Lebanon, Russian Federation, Taiwan, and United Arab Emirates. Int J Clin Pract 2014;68:1152-1160.

12) Donnelly C, Bangs M, Trzepacz P, Jin L, Zhang S, Witte MM, et al. Safety and tolerability of atomoxetine over 3 to 4 years in children and adolescents with ADHD. J Am Acad Child Adolesc Psychiatry 2009;48:176-185.

13) Gajria K, Lu M, Sikirica V, Greven P, Zhong $Y$, Qin P, et al. Adherence, persistence, and medication discontinuation in patients with attention-deficit/hyperactivity disorder - a systematic literature review. Neuropsychiatr Dis Treat 2014;10:1543-1569.

14) American Psychiatric Association. Diagnostic and Statistical Manual for Mental Disorder IV (DSM-IV). 4th ed. Washington DC: American Psychiatric Association;1994.

15) Kaufman J, Birmaher B, Brent D, Rao U, Flynn C, Moreci P, et al. Schedule for affective disorders and schizophrenia for school-age children-present and lifetime version (K-SADS-PL): initial reliability and validity data. J Am Acad Child Adolesc Psychiatry 1997;36: 980-988.

16) DuPaul GJ. Parent and teacher ratings of ADHD symptoms: psychometric properties in a community-based sample. J Clin Child Adolesc Psychol 1991;20:245-253.

17) Shin MS, Cho SZ, Chun SY, Hong KE. A study of the development and standardization of ADHD diagnostic system. Korean J Acad Child Adolesc Psychiatry 2000;11:91-99.

18) Guy W. Clinical global impressions. In: Guy W, editor. ECDEU assessment manual for psychopharmacology-revised. Rockville, MD: US Department of Health, Education, and Welfare;1976. p.217-222.

19) Cho SM, Park HY, Kim JH, Hong CH, Hwang ST. A standardization study of the Korean personality rating scale for children (KPRC). Korean J Clin Psychol 2006;25:825-848.

20) Lee MS, Lee SI, Hong SD, Kim JH, Choi J, Joung YS. Two different solicitation methods for obtaining information on adverse events associated with methylphenidate in adolescents: a 12-week multicenter, open-label study. J Child Adolesc Psychopharmacol 2013; 23:22-27.

21) Kim YS, Cheon KA, Kim BN, Chang SA, Yoo HJ, Kim JW, et al. The reliability and validity of kiddie-schedule for affective disorders and schizophrenia-present and lifetime version-Korean version (K-SADS-PL-K). Yonsei Med J 2004;45:81-89.

22) Park H, Kwak K, Park G. K-WPPSI manual. Seoul: Special Education;1995.

23) Kwak K, Park H, Kim C. Korean Wechsler intelligence scale for children-III (K-WISC-III). Seoul: Seoul Special Education Publishing $\mathrm{Co} ; 2001$.

24) Kwak K, Oh S, Kim C. Korean-Wechsler intelligence scale for chil-
dren-IV (K-WISC-IV). Seoul: Hakjisa;2011.

25) So YK, Noh JS, Kim YS, Ko SG, Koh YJ. The reliability and validity of Korean parent and teacher ADHD rating scale. J Korean Neuropsychiatr Assoc 2002;41:283-289.

26) Kim ST, Kim JJ, Song, DH, Lee HK, Joo YH, Hong CH. Manual of KPI-C. Seoul: Korea Guidance; 1997.

27) Perwien AR, Kratochvil CJ, Faries DE, Vaughan BS, Spencer T, Brown RT. Atomoxetine treatment in children and adolescents with attention-deficit hyperactivity disorder: what are the long-term health-related quality-of-life outcomes? J Child Adolesc Psychopharmacol 2006;16:713-724.

28) Lee SH, Song DH, Kim BN, Joung YS, Ha EH, Cheon KA, et al. Variability of response time as a predictor of methylphenidate treatment response in Korean children with attention deficit hyperactivity disorder. Yonsei Med J 2009;50:650-655.

29) Faedda GL, Baldessarini RJ, Glovinsky IP, Austin NB. Treatmentemergent mania in pediatric bipolar disorder: a retrospective case review. J Affect Disord 2004;82:149-158.

30) Van der Oord S, Prins PJ, Oosterlaan J, Emmelkamp PM. Treatment of attention deficit hyperactivity disorder in children. Predictors of treatment outcome. Eur Child Adolesc Psychiatry 2008; 17:73-81.

31) Mazzone L, Reale L, Mannino V, Cocuzza M, Vitiello B. Lower IQ is associated with decreased clinical response to atomoxetine in children and adolescents with attention-deficit hyperactivity disorder. CNS Drugs 2011;25:503-509.

32) Hechtman L. Predictors of long-term outcome in children with attention-deficit/hyperactivity disorder. Pediatr Clin North Am 1999; 46:1039-1052.

33) Swanson JM, Hinshaw SP, Arnold LE, Gibbons RD, Marcus S, Hur $\mathbf{K}$, et al. Secondary evaluations of MTA 36-month outcomes: propensity score and growth mixture model analyses. J Am Acad Child Adolesc Psychiatry 2007;46:1003-1014.

34) Henderson TA, Hartman K. Aggression, mania, and hypomania induction associated with atomoxetine. Pediatrics 2004;114:895-896.

35) Polzer J, Bangs ME, Zhang S, Dellva MA, Tauscher-Wisniewski S, Acharya N, et al. Meta-analysis of aggression or hostility events in randomized, controlled clinical trials of atomoxetine for ADHD. Biol Psychiatry 2007;61:713-719.

36) Guney E, Uneri OS. Atomoxetine-induced hypomania-like symptoms in a preadolescent patient. J Child Adolesc Psychopharmacol 2014;24:530-531.

37) Michelson D, Faries D, Wernicke J, Kelsey D, Kendrick K, Sallee FR, et al. Atomoxetine in the treatment of children and adolescents with attention-deficit/hyperactivity disorder: a randomized, placebo-controlled, dose-response study. Pediatrics 2001;108:E83.

38) Trzepacz PT, Williams DW, Feldman PD, Wrishko RE, Witcher JW, Buitelaar JK. CYP2D6 metabolizer status and atomoxetine dosing in children and adolescents with ADHD. Eur Neuropsychopharmacol 2008;18:79-86.

39) Yoon W, Park KJ, Kweon K, Kim HW. Clinical application of the Korean personality rating scale for children in attention-deficit hyperactivity disorder. J Korean Acad Child Adolesc Psychiatry 2015; 26:217-225.

40) Coghill DR, Joseph A, Sikirica V, Kosinski M, Bliss C, Huss M. Correlations between clinical trial outcomes based on symptoms, functional impairments, and quality of life in children and adolescents with ADHD. J Atten Disord. In press 2017. 